\title{
Budget Impact Analysis of Antiepileptic Drugs for Lennox-Gastaut Syndrome
}

\author{
Michelle Skornicki, MPH; Karen M. Clements, ScD; and Amy K. O'Sullivan, PhD
}

\begin{abstract}
BACKGROUND: In October 2011, clobazam was FDA-approved for adjunctive treatment of seizures associated with Lennox-Gastaut syndrome (LGS), a debilitating childhood epilepsy characterized by drop attacks, for patients 2 years and older.
\end{abstract}

OBJECTIVE: To assess the budget impact of adding clobazam to an antiepileptic drug (AED) portfolio containing topiramate, lamotrigine, and rufinamide in a hypothetical, 100,000-member commercially insured health plan.

METHODS: Patient characteristics and AED efficacy (decrease in dropseizure frequency) were modeled with clinical data. Medical costs were derived from administrative claims data from a large U.S. managed health plan, with the assumption that $2.3 \%$ of drop seizures required medical care. Two-year budget impact was measured. Results were expressed as the overall difference in costs (medical and pharmacy) to a health plan and cost per member per month (PMPM) after addition of clobazam. Analyses of alternative scenarios were performed.

RESULTS: With the assumption that $0.04 \%$ of the plan population had LGS, adding clobazam to the formulary resulted in cost savings of $\$ 98,059$ in year 1 and $\$ 131,690$ in year 2 (savings of $\$ 0.08$ and \$0.11 PMPM, respectively). Analyses of alternative scenarios with lower seizure rates upon discontinuation or greater long-term efficacy for lamotrigine and topiramate did not substantially alter conclusions. The assumption that fewer drop seizures required medical care resulted in a savings of approximately $\$ 5,000$ per year with clobazam, which suggested that medically attended drop seizures drive costs.

CONCLUSIONS: Medically attended drop seizures are a major cost driver for LGS patients. Adding clobazam to a health plan formulary can have a positive overall budget impact through decreased medical costs associated with drop seizures.

J Manag Care Pharm. 2014;20(4):400-06

Copyright $\odot 2014$, Academy of Managed Care Pharmacy. All rights reserved.

\section{What is already known about this subject}

- Lennox-Gastaut syndrome (LGS) is a severe, intractable syndrome of encephalopathy.

- Clobazam (Onfi) was approved in October 2011 as an adjunctive treatment for seizures associated with LGS for patients 2 years and older and has demonstrated substantial efficacy in the treatment of LGS in phase II and III randomized controlled trials.

- Information on the economic utility of the 5 antiepileptic drugs approved in the United States for adjunctive treatment of LGSclobazam, felbamate, lamotrigine, topiramate, and rufinamideis limited.

\section{What this study adds}

- This is the first study to develop and present a trial-based, budget impact model evaluating the addition of clobazam to a formulary plan.

- Medically attended drop seizures drive costs of caring for LGS patients.

- Adding clobazam to a health plan formulary can have a positive overall budget impact through decreased medical costs associated with drop seizures.

$\mathrm{L}$ ennox-Gastaut syndrome (LGS) is a severe encephalopathy that occurs in an estimated 1\%-10\% of childhood epilepsies, with onset typically occurring between the ages of 1 and 7 years. ${ }^{1,2}$ LGS is generally characterized by a triad of features that include multiple seizure types (e.g., tonic, myoclonic, atypical absences, sudden tonic, or atonic falls ["drop attacks"]) associated with frequent occurrence of nonconvulsive status epilepticus, the presence of slow spikeand-waves with paroxysmal fast rhythms in the electroencephalogram (EEG), and cognitive impairment. While drop attacks occur in at least $50 \%$ of patients with LGS, they are not diagnostic for the disease and are observed in other epilepsy syndromes. ${ }^{3}$ However, since none of these characteristics are pathognomonic, diagnosis of LGS requires a careful evaluation of clinical symptoms and the EEG. ${ }^{3}$

The prognosis for LGS is poor. Patients with this disorder have an increased risk of mortality, ${ }^{4,5}$ often resulting from seizure-related accidents. ${ }^{3}$ Approximately $50 \%$ of LGS patients experience sudden drop seizures that can lead to serious physical injury. ${ }^{1}$ These types of seizures are generally intractable and may require protective headgear, hindering a child's ability to participate in daily activities and engage in social interactions. In addition, cognitive impairment is found in approximately $20 \%-60 \%$ of patients at onset, with $75 \%-95 \%$ of patients demonstrating deterioration of function within 5 years of onset. ${ }^{1}$ Cognitive impairment is often associated with such behavioral problems as hyperactivity and aggressiveness, which may further limit a child's ability to attend school and participate in social activities. ${ }^{6}$ Together, the physical, cognitive, behavioral, and social challenges of LGS have a substantial negative impact on patients' quality of life and present a significant burden to their caregivers. ${ }^{7}$ By adulthood, most LGS patients continue to require assistance and are unable to live independently., 
In addition to the personal challenges faced by patients and their families, childhood epilepsies such as LGS present a considerable economic burden. Factors to consider when evaluating the economic impact of these disorders include the costs of diagnostic tests, medical services, referrals to specialists, medications, social services for children, and missed work or underemployment for caregivers. ${ }^{9}$ Analyses based on the Kids' Inpatient Databases found that seizures accounted for 16.9\% of hospital admissions in 2006, ${ }^{10}$ with an estimated annual cost per patient of $\$ 4,553 .{ }^{11}$ These results were consistent with a survey-based economic analysis, which found that annual U.S. medical costs were significantly greater for children with epilepsy ( $\$ 6,379$, adjusted to 2004 prices) than for children without epilepsy $(\$ 1,976) .^{12}$ This study also found that for both children and adults, patients with epilepsy were more likely to be uninsured or only have public insurance, which may pose a serious societal burden given the substantial total costs of treating epilepsy. In another survey-based analysis of adult patients, the projected annual cost of epilepsy to society was US $\$ 12.5$ billion, including direct costs (e.g., medical services, diagnostic procedures, emergency room visits, treatment) and indirect costs (e.g., lost wages), adjusted to 1995 prices. ${ }^{13}$ The vast majority of these costs (79.5\%) were attributed to patients who had intractable seizures, highlighting the economic burden of LGS and other severe forms of epilepsy. While LGS is generally a syndrome of intractable seizures, not all intractable seizure disorders are LGS.

Seizure reduction is one of the main treatment goals in LGS, and patients often require several concomitant medications because of the refractory nature of their seizures. Currently, 5 antiepileptic drugs (AEDs) are approved by the U.S. Food and Drug Administration (FDA) for the treatment of LGS: clobazam, felbamate, lamotrigine, topiramate, and rufinamide. Clonazepam is also approved for LGS, but there are no published randomized clinical studies on its use in the disease. Clobazam (Onfi) was approved in October 2011 as an adjunctive treatment for seizures associated with LGS for patients 2 years and older. Clobazam has demonstrated substantial efficacy in the treatment of LGS in phase II and III randomized controlled trials. In an indirect comparison of clobazam with other LGS therapies via transformation of clinical trial primary endpoints into Cohen's d effect sizes, high- and mediumdosage clobazam was estimated to be more efficacious than felbamate, lamotrigine, topiramate, and rufinamide..$^{14}$

Available information about the economic utility of these AEDs in LGS, however, is very limited, although recent analyses have reported the cost-effectiveness of rufinamide, based on reduced frequency of total seizures and drop attacks ${ }^{15}$ and lower incremental costs per quality-adjusted life-years. ${ }^{16}$ With the recent U.S. approval of clobazam as an adjunctive treatment in LGS, the current analysis was conducted to estimate the budget impact of including clobazam as a treatment option in a hypothetical commercial health care plan, based on dropseizure results from published LGS clinical trials.

\section{Methods}

\section{Model Overview}

A trial-based economic model was constructed to evaluate the budget impact of adding clobazam to a portfolio of other AEDs approved for the treatment of LGS. Three modules were developed to construct this budget impact model: (1) patient population module, used to estimate the percentage of patients in a hypothetical, 100,000-member health care plan who would be eligible for treatment with clobazam; (2) marketplace dynamics module, which took into account current and future market shares for LGS therapies over a 2-year period; and (3) cost module, which estimated the total costs of treating patients prior to and after adding clobazam to the hypothetical formulary, and compared these costs with other therapies.

\section{Patient Model Population and AED Comparators}

The target population comprised individuals with LGS who had characteristics that were similar to patients in the pivotal clobazam trial OV-1012 (also known as the CONTAIN trial). ${ }^{17}$ Key inclusion criteria for this study were as follows: (a) children and adults ages 2 to 54 years with onset of LGS before 11 years of age, (b) $\geq 2$ drop seizures per week, and (c) concomitant therapy with 1 to 3 other adjunctive AEDs. Key exclusion criteria in CONTAIN included (a) previous treatment with clobazam, (b) concurrent treatment with long-term systemic steroid therapy, and (c) episode of status epilepticus within 12 weeks of baseline.

With published data from separate trials on their use as adjunctive therapy for LGS, the 3 comparators used in the model were rufinamide (maximum dosage, 45 milligrams per kilograms per day $[\mathrm{mg} / \mathrm{kg} /$ day]), topiramate (target dosage, $6 \mathrm{mg} / \mathrm{kg} /$ day), and lamotrigine (maximum dosage, $5 \mathrm{mg} / \mathrm{kg} /$ day for patients receiving concomitant valproate or $15 \mathrm{mg} /$ $\mathrm{kg} / \mathrm{day}$ for patients not receiving valproate). These AEDs have been approved by the FDA for LGS based on clinical trials ${ }^{18-20}$ that included patients with characteristics similar to those of patients in the CONTAIN trial (i.e., age range, number of drop seizures, seizures not controlled by previous regimens, use of study drug therapy as adjunctive therapy). Felbamate was excluded, as none of its studies were conducted with patients of characteristics similar to those in the clobazam, rufinamide, topiramate, and lamotrigine trials. Clonazepam was also excluded, as there are no published randomized clinical studies on its use in LGS.

\section{Model Structure and Inputs}

This budget impact model was developed to calculate the number of drop seizures occurring over 2 years with clobazam and each of the comparator treatments. The 2-year time 


\begin{tabular}{|c|c|c|}
\hline Input & \multicolumn{2}{|c|}{ Value } \\
\hline \multicolumn{3}{|l|}{ Model population } \\
\hline Health plan population, $\mathrm{n}^{\mathrm{a}}$ & \multicolumn{2}{|c|}{100,000} \\
\hline Population with LGS, \%b & \multicolumn{2}{|c|}{0.04} \\
\hline LGS patients eligible for treatment, $\%^{\mathrm{a}}$ & \multicolumn{2}{|c|}{100} \\
\hline \multicolumn{3}{|l|}{ Marketplace dynamics ${ }^{c}$} \\
\hline \multicolumn{3}{|l|}{ Without clobazam, \% global market share } \\
\hline Clobazam & \multicolumn{2}{|c|}{0} \\
\hline Rufinamide & \multicolumn{2}{|c|}{9} \\
\hline Topiramate & \multicolumn{2}{|c|}{42} \\
\hline Lamotrigine & \multicolumn{2}{|c|}{49} \\
\hline \multicolumn{3}{|l|}{ Wholesale acquisition costs (2013) } \\
\hline Clobazam (\$) & \multicolumn{2}{|c|}{22.41} \\
\hline Rufinamide (\$) & \multicolumn{2}{|c|}{31.69} \\
\hline Topiramate (\$) & \multicolumn{2}{|c|}{0.12} \\
\hline Lamotrigine (\$) & \multicolumn{2}{|c|}{0.12} \\
\hline With clobazam, \% global market share & Model Year 1 & Model Year 2 \\
\hline Clobazam & 7 & 11 \\
\hline Rufinamide & 8 & 8 \\
\hline Topiramate & 39 & 37 \\
\hline Lamotrigine & 46 & 44 \\
\hline \multicolumn{3}{|c|}{$\begin{array}{l}{ }^{a} \text { Model assumption. } \\
{ }^{b} \text { Based on published estimates. }{ }^{2,21} \\
{ }^{c} \text { Based on market research (data on file, Lundbeck LLC). } \\
\text { LGS = Lennox-Gastaut syndrome. }\end{array}$} \\
\hline
\end{tabular}

horizon was chosen to reflect the budget cycles of U.S. commercial payers.

Of the 100,000 members included in the hypothetical health care plan, $0.04 \%$ were assumed to have had an LGS diagnosis (Table 1) based on the midpoint of the range of prevalence estimates of LGS in the U.S. population., ${ }^{2,21}$ All LGS patients in the model were considered eligible for clobazam treatment, and the number of patients eligible for treatment for LGS was assumed to have been constant over the 2-year time horizon. Marketplace dynamics were estimated based on market research data, including current market shares for model comparators and projected market shares for clobazam (Table 1).

The average number of drop seizures per patient was estimated to have been 132 seizures per month, ${ }^{22}$ based on baseline data from the open-label, long-term extension of the CONTAIN trial. ${ }^{23}$ During each month of the model, patients were categorized as follows based on their percentage decreases in drop seizures from baseline: 0 to $<50 \%$ (nonresponse), $50 \%$ to $<75 \%, 75 \%$ to $<100 \%$, and $100 \%$ (seizure-free). Patients in each category were assumed to have had the midpoint number of seizures in their categories, and changes in drop seizures were assumed to have been linear between data points.

Data from open-label studies of clobazam ${ }^{23}$ and rufinamide $^{24}$ were employed to estimate mean changes in drop-
TABLE 2 Drop-Seizure Decreases Following Treatment with Clobazam and Rufinamide After 24 Months of Treatment

\begin{tabular}{l|c|c}
\hline & Clobazam & Rufinamide \\
\hline \multicolumn{3}{|c|}{ Percentage decrease in drop-seizure category, \% of patients } \\
\hline$<50 \%$ & 47 & 71 \\
\hline $50 \%$ to $<75 \%$ & 2 & 7 \\
\hline $75 \%$ to $<100 \%$ & 24 & 16 \\
\hline $100 \%$ (seizure-free) & 27 & 6 \\
\hline Mean percentage decrease & 52 & 33 \\
\hline
\end{tabular}

seizure frequency over 2 years of treatment with these medications (Table 2). Because the percentages of rufinamide-treated patients in the drop-seizure reduction categories were reported only at 36 months, interim efficacy was calculated via several assumptions. Based on published data presenting median reduction in drop seizures over 36 months, ${ }^{24}$ the fraction of the 36-month median reduction achieved at each 3-month interval was calculated. These fractions were then multiplied by the percentage of patients in each drop-seizure reduction category at the end of the trial to obtain the number of patients in each reduction category at every 3 months. Rufinamide efficacy through 24 months was used for consistency with the available clobazam data.

Estimates of drop-seizure reduction were only available at 3 months for lamotrigine and at $\geq 3$ and $\geq 6$ months for topiramate. ${ }^{19,20,25}$ We assumed that the efficacies of these comparators after 3 months of treatment followed the same trends found for clobazam treatment. Relative clobazam efficacy (i.e., mean reduction in drop seizures) was calculated for 12 months versus 3 months $(64.1 \% / 62.1 \%=1.03)$, and for 24 months versus 3 months $(52.4 \% / 62.1 \%=0.83)$. These relative reduction estimates were multiplied by 3 -month data to derive 12- and 24-month mean drop-seizure reduction rates for topiramate (12 months, 47\%; 24 months, 39\%) and lamotrigine (12 months, $41 \%$; 24 months, 34\%). For all LGS treatments included in the model, change in drop seizures was assumed to have been linear between data points.

Over 2 years, we estimated that $17.9 \%$ of patients receiving clobazam and $57.3 \%$ of patients receiving rufinamide discontinued treatment. Comparable published rates are 20\% (interim analysis of long-term, open-label study of clobazam $^{23}$ ) and 66\% (36-month, open-label study of rufinamide ${ }^{24}$, respectively. We assumed that after treatment discontinuation, patients returned to baseline levels of drop seizures. For lamotrigine and topiramate, long-term discontinuation relative to short-term discontinuation was assumed to have been the same as for clobazam. Of note, the published article on the open-label extension of topiramate does not provide estimates of discontinuation at 2 years. ${ }^{25}$ 

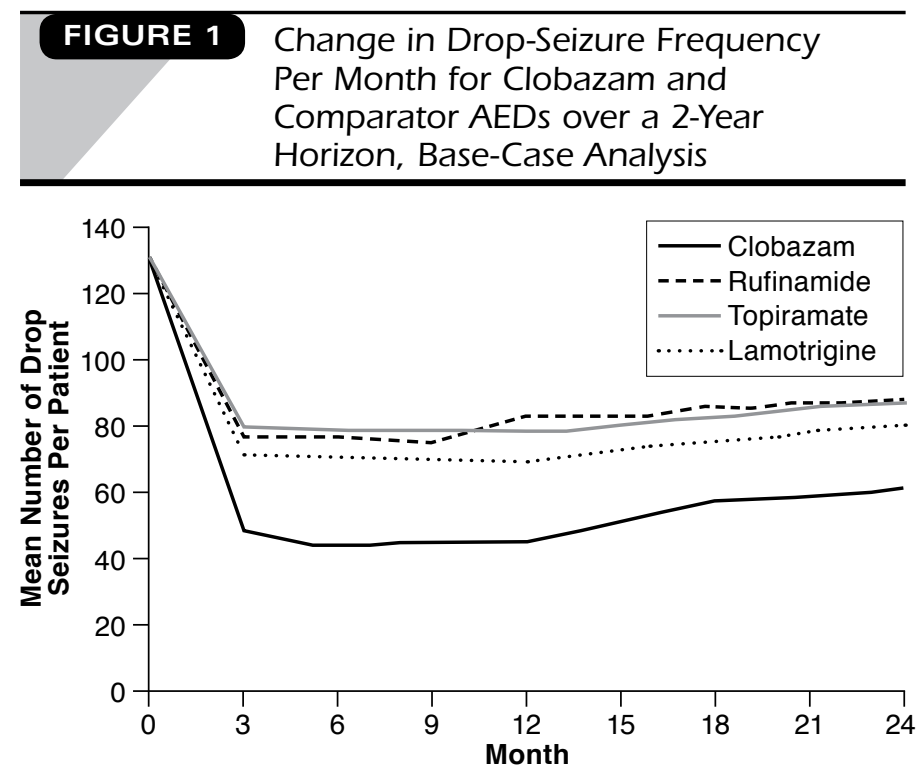

AEDs = antiepileptic drugs.

\section{Estimated Costs}

Drug costs, presented in U.S. dollars, were based on published wholesale acquisition costs for $2013^{26}$ (Table 1) and average dosages reported in LGS trials. ${ }^{17-20}$ Medical costs were estimated in a retrospective cohort analysis of claims data from a large managed health care plan. ${ }^{27}$ Plan participants who had International Classification of Diseases, Ninth Revision, Clinical Modification (ICD-9-CM) codes for epilepsy ( $\geq 2$ claims) and developmental delay ( $\geq 1$ claim) from January 1, 2007, to September 30, 2009, were identified as LGS patients and included in medical cost analyses. We assumed the background costs of concomitant AEDs were similar for all therapies.

Medically attended seizures were defined as claims that had a diagnosis or procedure code for "seizure-attributable event." A seizure was defined as any observed medical claim with ICD9-CM diagnosis code 345.0-345.9 in any position. One seizure per day was counted. ${ }^{22}$ Costs per seizure were calculated based on the following criteria: (a) for mild events (e.g., lacerations, contusions), medical claims occurring within 10 days following the initial observed claim; (b) for moderate events (e.g., closed fractures excluding head fractures, sprains), claims occurring within 30 days following the initial claim; and (c) for severe events (e.g., head injury, closed or open skull fractures, cortex [cerebral] lacerations), claims occurring within 90 days following the initial claim. Total medical costs included ambulatory services, emergency room, and hospital inpatient costs. An estimate of the percentage of medically attended drop seizures could not be identified directly from the literature or database analysis but was determined from the results of a survey of

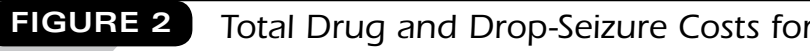 the World Without Clobazam Versus the World with Clobazam, Base-Case Analysis $^{\mathrm{a}}$}

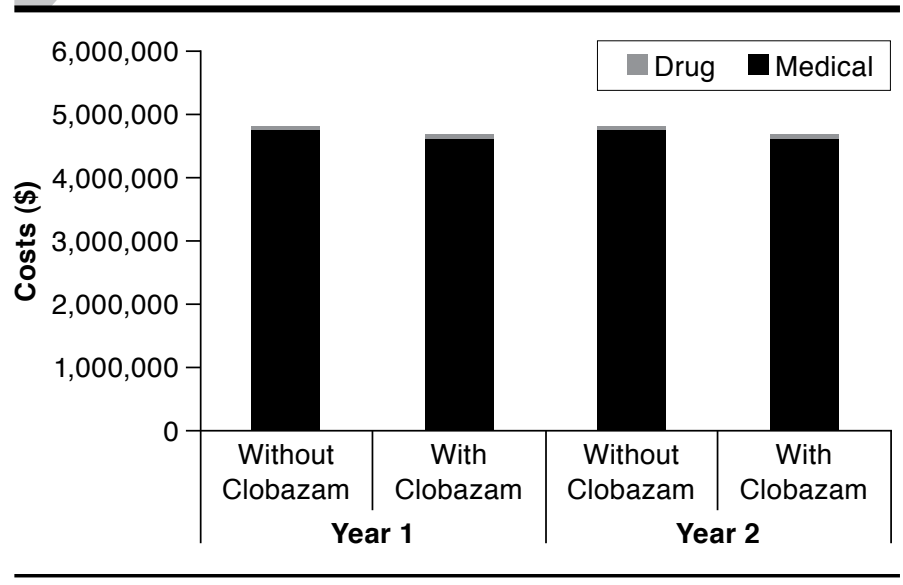

aTotal medical costs included ambulatory services, emergency room, and hospital inpatient costs

physicians in the United Kingdom of the percentage of seizures that required hospitalization and a U.S.-based retrospective claims database analysis. ${ }^{16,22}$ From the claims database analysis and literature estimates, $0.5 \%$ of drop attacks were assumed to result in hospitalization, representing $21.5 \%$ of all seizures requiring any medical attention. With these assumptions, 2.3\% of drop seizures were assumed to be medically attended, and the mean total medical cost per seizure-attributable eventadjusted for inflation to 2013 prices using the annual medical care component of the Consumer Price Index-was \$5,501.

\section{Budget Impact Analysis}

The budget impact of clobazam was estimated as the difference in net costs between 2 scenarios: (a) a scenario in which the LGS patient population is treated with comparator medications; and (b) a scenario in which clobazam becomes available as a treatment option. Analyses included total costs, costs by component (i.e., pharmacy, direct medical), and per-member per-month (PMPM) costs.

Base-case analyses were performed from the perspective of a private health care payer, such as a commercial health insurer. In addition, when underlying model assumptions were uncertain, conservative alternative-scenario analyses were conducted to test the impact of alternative plausible assumptions. The following 4 alternative scenarios were conducted:

1. Seizure retention after treatment discontinuation. While the base case was evaluated with the assumption that discontinuers returned to their baseline seizure rates, an alternative scenario evaluated results with the assumption that LGS patients who discontinued treatment were assumed 
Budget Impact Analysis of Antiepileptic Drugs for Lennox-Gastaut Syndrome

\section{TABLE 3 Clobazam Budget Impact, Base-Case, and Alternative Analyses}

\begin{tabular}{|c|c|c|c|c|c|c|c|c|c|c|}
\hline \multirow[b]{2}{*}{ Assumption } & \multicolumn{2}{|c|}{ Base Case } & \multicolumn{2}{|c|}{ Alternative Scenario $1^{\mathrm{a}}$} & \multicolumn{2}{|c|}{ Alternative Scenario $2^{b}$} & \multicolumn{2}{|c|}{ Alternative Scenario $3^{c}$} & \multicolumn{2}{|c|}{ Alternative Scenario $4^{\mathrm{d}}$} \\
\hline & Year 1 (\$) & Year $2(\$)$ & Year 1 (\$) & Year $2(\$)$ & Year 1 (\$) & Year 2 (\$) & Year 1 (\$) & Year 2 (\$) & Year 1 (\$) & Year $2(\$)$ \\
\hline \multicolumn{11}{|l|}{ Cost without } \\
\hline Clobazam & $4,796,755$ & $4,820,553$ & $4,633,454$ & $3,986,924$ & $4,796,755$ & $4,420,918$ & $1,059,183$ & $1,054,464$ & $1,229,937$ & $1,236,039$ \\
\hline \multicolumn{11}{|l|}{ Cost with } \\
\hline Clobazam & $4,698,696$ & $4,688,863$ & $4,535,940$ & $3,831,836$ & $4,698,696$ & $4,334,092$ & $1,054,064$ & $1,048,698$ & $1,204,794$ & $1,202,273$ \\
\hline Totale & $(98,059)$ & $(131,690)$ & $(97,514)$ & $(155,088)$ & $(98,059)$ & $(86,826)$ & $(5,119)$ & $(5,766)$ & $(25,143)$ & $(33,767)$ \\
\hline Cost/PMPMe & $(0.08)$ & $(0.11)$ & $(0.08)$ & $(0.13)$ & $(0.08)$ & $(0.07)$ & 0.00 & 0.00 & $(0.02)$ & $(0.03)$ \\
\hline \multicolumn{11}{|c|}{$\begin{array}{l}\text { aAssumed that patients who stop treatment retain the same level of drop seizz } \\
\text { bAssumed that seizure reduction with topiramate and lamotrigine continues } \\
\text { 'Assumed that only } 0.5 \% \text { of drop seizures are medically attended. } \\
\text { dAssumed that only } 0.01 \% \text { of the population is eligible to receive clobazam. } \\
\text { In U.S. dollars; parentheses indicate savings. } \\
\text { PMPM = per patient per month. }\end{array}$} \\
\hline
\end{tabular}

to have retained their average percentage reductions in drop seizures for the remainder of the 2-year horizon.

2. Long-term efficacy of lamotrigine and topiramate. Base-case analyses assumed efficacy of lamotrigine and topiramate declined in the second year, as with patients receiving clobazam. An alternative scenario was conducted with the assumption that patients receiving lamotrigine and topiramate continued experiencing a $3 \%$ increase in seizure reduction during year 2, as was estimated for months 3 to 12

3. Percentage of seizures that require medical attention. Because the percentage of drop seizures that require medical attention could not be assessed directly from the literature or database analyses, we evaluated a more conservative assumption that only $0.5 \%$ of drop seizures were medically attended and incurred treatment costs (the estimate of the percentage of drop seizures requiring hospitalization reported by Verdian et al. 2010'6).

4. Percentage of population eligible for clobazam. While the base case used a midpoint estimate of a range of estimates of prevalence of LGS, the alternative scenario evaluated the lower end of the range, with the assumption that only $0.01 \%$ of the plan population had been diagnosed with LGS and were eligible for clobazam.

\section{Results}

\section{Drop Seizures}

The mean number of drop seizures per month decreased for clobazam and AED comparators over the 2-year time horizon used in this model (Figure 1). At 12 and 24 months, respectively, the estimated percentage reductions in drop seizures were clobazam, 64\% and 52\%; rufinamide, 37\% and 33\%; topiramate, $47 \%$ and $39 \%$; and lamotrigine, $41 \%$ and $34 \%$.

\section{Budget Impact}

In years 1 and 2 of the budget impact model, total costs (i.e., drug and medical costs) were lower in the future scenario with clobazam than in the current scenario without clobazam (Figure 2). The overwhelming majority of costs in all scenarios were the treatment of drop seizures (range: 91.9\%-99.9\%). In this base-case analysis, the inclusion of clobazam as a treatment option resulted in lower total costs (savings of $\$ 98,059$ in year 1 and $\$ 131,690$ in year 2 ) and savings PMPM (\$0.08 in year $1, \$ 0.11$ in year 2; Table 3).

Results from analyses with alternative scenarios 1 and 2 were generally similar to those found in the base-case analysis (Table 3), with a few notable differences. When treatment discontinuers were assumed to have retained the same levels of drop-seizure reduction that they had achieved when treatment was stopped, the PMPM savings in year 2 was slightly greater $(\$ 0.13)$ than in the base-case analysis $(\$ 0.11)$. When continued increases in the reduction of drop seizures were assumed to have followed in year 2 for topiramate and lamotrigine, the PMPM savings during that period was less $(\$ 0.07)$ than in the base-case analysis.

With the assumption that only $0.5 \%$ of drop seizures were medically attended (alternative scenario 3), the total estimated costs in year 1 (current, $\$ 1,059,183$; future, $\$ 1,054,064$ ) and year 2 (current, $\$ 1,054,464$; future, $\$ 1,048,698$ ) were less than overall costs found in the base-case analysis. However, no PMPM savings $(\$ 0.00)$ were found in year 1 or year 2 (Table 3).

With the assumption that only $0.01 \%$ of a plan population had LGS and were eligible for treatment (alternative scenario $4)$, estimated savings in year $1(\$ 25,143)$ and year $2(\$ 33,767)$ were less than overall costs in the base case. In addition, PMPM savings were correspondingly lower ( $\$ 0.02$ in year $1, \$ 0.03$ in year 2). 


\section{Discussion}

In this budget impact analysis, a hypothetical, 100,000-member health care plan model was developed to evaluate the costs of including clobazam as a treatment option for LGS. Results from the base-case analysis indicated that over a 2-year time horizon, inclusion of clobazam as a treatment option resulted in savings for mean total costs and PMPM costs, with greater savings in year 2 than year 1 . Similar results were found in an alternative scenario in which it was assumed that treatment discontinuers had retained the same numbers of drop seizures as they had when they stopped receiving medication (rather than returning to baseline levels). In a scenario with improved long-term efficacy, the cost savings in year 2 was similar to results from the base-case analysis (\$0.11).

Since no published long-term studies with lamotrigine and topiramate for the treatment of LGS were available, it was assumed in the base-case analysis that long-term efficacy was relative to short-term efficacy in a manner similar to the longand short-term efficacy results found with clobazam. In this assumption, mean seizure reduction improved by $3 \%$ from 3 to 12 months of treatment and then declined by $17 \%$ during the second year of treatment. Thus, an alternative scenario 2 was developed that assumed that mean seizure reduction continued to improve by $3 \%$ during year 2 of the model. In this alternative scenario, cost savings associated with clobazam were lower during year 2 (PMPM, \$0.06) than was found in the base-case analysis (PMPM, \$0.11).

The base-case analysis assumption that $2.3 \%$ of all drop seizures in the hypothetical health care plan population received medical attention was based on published literature and a retrospective analysis of claims data. ${ }^{27}$ Interpretation of the claims analysis is limited, since there is no ICD-9-CM code specific to LGS, and the algorithm used to identify the patient population has not been validated. Furthermore, claims data are subject to coding errors, such as coding for the purpose of rule-out or undercoding, and certain clinical and disease-specific parameters that could affect study outcomes are not readily available. Finally, given that the study was conducted in a managed care population, the data may not be indicative of patients in a nonmanaged care setting.

As expected, the overall costs were lower than in the basecase analysis for an alternative scenario that assumed only $0.5 \%$ of drop seizures received medical attention. However, negligible cost savings were found during the 2-year time horizon. These results suggest that managing seizures in patients with LGS is not just a clinical issue. Medically attended drop seizures are major drivers of health care costs in this population, and adequate treatment of these events may have economic benefits. Budget impact results are dependent on the number of LGS patients in a plan's population. As estimates of the prevalence of LGS vary widely from $1 \%$ to $10 \%$ of all epilepsies, we evaluated a more conservative estimate at the lower end of the range and still found more than $\$ 25,000$ savings (PMPM $=\$ 0.02$ ).

\section{Limitations}

As with all models, the main limitation of this budget impact model was the simplification of assumptions required to compare a world with clobazam to a world without clobazam. For example, mean number of drop seizures was based on the assumption that patients in each category had the midpoint number of seizures for that category, and changes in mean drop seizures were assumed to have been linear during each 3-month interval. Other base-case assumptions have been previously discussed. In addition, applying the patient inclusion/ exclusion criteria from the phase III CONTAIN trial of clobazam to determine if LGS patients would be considered eligible for clobazam treatment means we may have overestimated the rate of drop seizures in the LGS population. However, several alternative scenarios were developed to evaluate how different factors might affect cost outcomes. In particular, we completed sensitivity analyses, including a scenerio with a lower number of drop seizures that require medical attention.

\section{Conclusions}

Overall, with the exception of an alternative scenario in which only very few drop seizures were medically attended, adding clobazam to the budget impact model resulted in reduced mean total costs as well as PMPM costs. These savings may be attributable to lower health care costs for patients receiving clobazam, resulting from greater reduction in medically attended drop seizures versus other treatment options.

\section{Authors}

MICHELLE SKORNICKI, MPH, was Analyst; KAREN M CLEMENTS, SCD, was Senior Manager; and AMY K. O'SULLIVAN, $\mathrm{PhD}$, was Director, Health Economics \& Outcomes Research, Life Sciences, OptumInsight, Medford, Massachusetts.

AUTHOR CORRESPONDENCE: Michelle Skornicki, MPH, formerly Analyst, Health Economics \& Outcomes Research, Life Sciences, OptumInsight, 10 Cabot Rd., Ste. 304, Medford, MA 02155.

E-mail: michelle.skornicki@gmail.com.

\section{DISCLOSURES}

This study was fully funded by Lundbeck LLC, Deerfield, Illinois. The analyses reported in this manuscript were performed by OptumInsight, Medford, Massachusetts, and funded through a contract with Lundbeck LLC. Clements, Skornicki, and O'Sullivan were employees of OptumInsight at the time the analyses were conducted.

Study concept and design were contributed by Skornicki, O'Sullivan, and Clements. Data was collected primarily by Skornicki, with assistance from O'Sullivan and Clements, and interpreted by Skornicki, Clements, and O'Sullivan. The manuscript was drafted by Mildred Bahn of Prescott Medical Communications Group at the direction of the authors, and all authors worked on the revision. 


\section{ACKNOWLEDGMENTS}

Writing assistance and editorial support during manuscript preparation and revision were provided by Mildred Bahn of Prescott Medical Communications Group, Chicago, Illinois, and Michael A. Nissen, ELS, Lundbeck LLC. This support was funded by Lundbeck LLC. The authors thank Jouko Isojarvi, MD, $\mathrm{PhD}$, and Cynthia Mueller, PhD, both of Lundbeck, for their critical reviews of this manuscript.

\section{REFERENCES}

1. Arzimanoglou A, French J, Blume WT, et al. Lennox-Gastaut syndrome: a consensus approach on diagnosis, assessment, management, and trial methodology. Lancet Neurol. 2009;8(1):82-93.

2. Camfield PR. Definition and natural history of Lennox-Gastaut syndrome. Epilepsia. 2011;52(Suppl 5):S3-S9.

3. Arzimanoglou A, Resnick T. All children who experience epileptic falls do not necessarily have Lennox-Gastaut syndrome ... but many do. Epileptic Disord. 2011;13(Suppl 1):S3-S13

4. Camfield C, Camfield P. Twenty years after childhood-onset symptomatic generalized epilepsy the social outcome is usually dependency or death: a population-based study. Dev Med Child Neurol. 2008;50(11):859-63.

5. Autry AR, Trevathan E, Van Naarden Braun K, Yeargin-Allsopp M. Increased risk of death among children with Lennox-Gastaut syndrome and infantile spasms. J Child Neurol. 2010;25(4):441-47.

6. Kerr M, Kluger G, Philip S. Evolution and management of Lennox-Gastaut syndrome through adolescence and into adulthood: are seizures always the primary issue? Epileptic Disord. 2011;13(Suppl 1):S15-S26.

7. Gallop K, Wild D, Nixon A, Verdian L, Cramer JA. Impact of LennoxGastaut Syndrome (LGS) on health-related quality of life (HRQL) of patients and caregivers: literature review. Seizure. 2009;18(8):554-58.

8. Camfield PR, Gibson PA, Douglass LM. Strategies for transitioning to adult care for youth with Lennox-Gastaut syndrome and related disorders. Epilepsia. 2011;52(Suppl 5):S21-S27.

9. Beghi E, Frigeni B, Beghi M, De Compadri P, Garattini L. A review of the costs of managing childhood epilepsy. PharmacoEconomics. 2005;23(1):27-45.

10. Berry JG, Poduri A, Bonkowsky JL, et al. Trends in resource utilization by children with neurological impairment in the United States inpatient health care system: a repeat cross-sectional study. PLoS Med. 2012:9(1):e1001158.

11. Standridge SM, Horn PS. Variations in hospitalization outcomes in children admitted with seizures between 2003 and 2006. J Child Neurol. 2012;27(7):898-906

12. Yoon D, Frick KD, Carr DA, Austin JK. Economic impact of epilepsy in the United States. Epilepsia. 2009;50(10):2186-91.
13. Begley CE, Famulari M, Annegers JF, et al. The cost of epilepsy in the United States: an estimate from population-based clinical and survey data. Epilepsia. 2000;41(3):342-51.

14. Cramer JA, Sapin C, François C. Indirect comparison of clobazam and other therapies for Lennox-Gastaut syndrome. Acta Neurol Scand. 2013;128(2):91-99.

15. Benedict A, Verdian L, Maclaine G. The cost effectiveness of rufinamide in the treatment of Lennox-Gastaut syndrome in the UK. Pharmacoeconomics. 2010;28(3):185-99.

16. Verdian L, Yi Y. Cost-utility analysis of rufinamide versus topiramate and lamotrigine for the treatment of children with Lennox-Gastaut Syndrome in the United Kingdom. Seizure. 2010;19(1):1-11.

17. Ng YT, Conry JA, Drummond R, et al. Randomized, phase III study results of clobazam in Lennox-Gastaut syndrome. Neurology. 2011;77(15):1473-81.

18. Glauser T, Kluger G, Sachdeo R, Krauss G, Perdomo C, Arroyo S. Rufinamide for generalized seizures associated with Lennox-Gastaut syndrome. Neurology. 2008;70(21):1950-58

19. Sachdeo RC, Glauser TA, Ritter F, Reife R, Lim P, Pledger G. A doubleblind, randomized trial of topiramate in Lennox-Gastaut syndrome. Topiramate YL Study Group. Neurology. 1999;52(9):1882-87.

20. Motte J, Trevathan E, Arvidsson JF, Barrera MN, Mullens EL, Manasco P. Lamotrigine for generalized seizures associated with the LennoxGastaut syndrome. Lamictal Lennox-Gastaut Study Group. N Engl J Med. 1997;337(25):1807-12.

21. Kobau R, Zahran H, Thurman DJ, et al. Epilepsy surveillance among adults-9 States, Behavioral Risk Factor Surveillance System, 2005. MMWR Surveill Summ. 2008;57(6):1-20.

22. Clements KM, Skornicki M, O'Sullivan AK. Cost-effectiveness analysis of antiepileptic drugs in the treatment of Lennox-Gastaut syndrome. Epilepsy Behav. 2013;29(1):184-89.

23. Ng YT, Conry J, Paolicchi J, et al. Long-term safety and efficacy of clobazam for Lennox-Gastaut syndrome: interim results of an open-label extension study. Epilepsy Behav. 2012;25(4):687-94.

24. Kluger G, Glauser T, Krauss G, Seeruthun R, Perdomo C, Arroyo S. Adjunctive rufinamide in Lennox-Gastaut syndrome: a long-term, openlabel extension study. Acta Neurol Scand. 2010;122(3):202-08.

25. Glauser TA, Levisohn PM, Ritter F, Sachdeo RC, et al. Topiramate in Lennox-Gastaut Syndrome: open-label treatment of patients completing a randomized controlled trial. Epilepsia. 2000;41(Suppl 1):S86-S90.

26. PDR Network. Red Book 2010: Pharmacy's Fundamental Reference. Montvale, NJ: Physicians' Desk Reference Incorporated, 2010.

27. Swindle JP, Song R, Liu F, et al. Economic burden of Lennox-Gastaut Syndrome [abstract]. Value Health. 2012;15(4):Al43. 